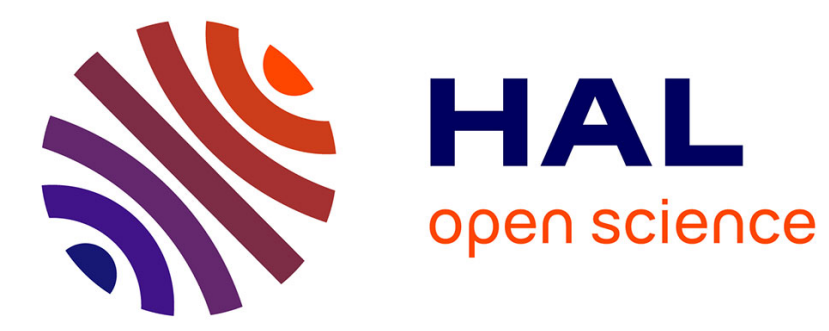

\title{
Carl G. Jung's Synchronicity and Quantum Entanglement: Schrödinger's Cat 'Wanders' Between Chromosomes
}

\author{
Igor V. Limar
}

\section{To cite this version:}

Igor V. Limar. Carl G. Jung's Synchronicity and Quantum Entanglement: Schrödinger's Cat 'Wanders' Between Chromosomes. NeuroQuantology, 2011, 9 (2), pp.313-321. hprints-00637383v3

HAL Id: hprints-00637383

https://hal-hprints.archives-ouvertes.fr/hprints-00637383v3

Submitted on 19 Jul 2012

HAL is a multi-disciplinary open access archive for the deposit and dissemination of scientific research documents, whether they are published or not. The documents may come from teaching and research institutions in France or abroad, or from public or private research centers.
L'archive ouverte pluridisciplinaire HAL, est destinée au dépôt et à la diffusion de documents scientifiques de niveau recherche, publiés ou non, émanant des établissements d'enseignement et de recherche français ou étrangers, des laboratoires publics ou privés. 


\title{
C.G. Jung's Synchronicity and Quantum Entanglement: Schrodinger's Cat 'Wanders' Between Chromosomes
}

\author{
Igor V. Limar
}

\begin{abstract}
One of the most prospective directions of study of C.G. Jung's synchronicity phenomenon is reviewed considering the latest achievements of modern science. The attention is focused mainly on the quantum entanglement and related phenomena - quantum coherence and quantum superposition. It is shown that the quantum non-locality capable of solving the Einstein-Podolsky-Rosen paradox represents one of the most adequate physical mechanisms in terms of conformity with the Jung's synchronicity hypothesis. An attempt is made on psychophysiological substantiation of synchronicity within the context of molecular biology. An original concept is proposed, stating that biological molecules involved in cell division during mitosis and meiosis, particularly DNA may be considered material carriers of consciousness. This assumption may be formulated on the basis of phenomenology of Jung's analytical psychology.
\end{abstract}

Key Words: consciousness, synchronicity, quantum entanglement, nucleic acids, mitosis, meiosis

The article had been published in the journal 'NeuroQuantology' - (2011) Volume 9, Issue 2, pp 313-321

\section{Link: http://www.neuroquantology.com/index.php/journal/article/view/376}

\author{
Problem formulation \\ related studies \\ To be sure, the concept of \\ synchronicity represents an integral part of \\ analytical psychology. Carl Jung believed, \\ that synchronicity is closely related to \\ numerous manifestations of psychic life of

\footnotetext{
Corresponding author: Igor V. Limar

Address: Institute of Innovative and Postgraduate Education (IIPE). Department of computer science and informational technologies, Dvoryanskaya str., 2, Odessa, 65026, Ukraine

Phone: +38 0487253687

Fax: -

e-mail: iv.limar@onu.edu.ua
}

the humans, both normal and affected by pathology. Determining the nature of the phenomenon of synchronicity may become important for psychotherapeutic practice, particularly for difficult clinical cases which today cannot be subjected to psychological correction using methods of classical psychiatry. At the same time, we know that exotic nature of 'manifestations of synchronicity' caused to certain extent skeptical attitude by a number of researchers. Considering criticism of Jung's theory of synchronicity by his opponents, it should be, nevertheless, admitted that in doing his research the founder of analytical 
psychology was guided, in particular, by principles of contemporary theoretical physics. For instance, it is well known that Jung has developed the concept of synchronicity in close collaboration with Wolfgang Pauli. Thus, the Swiss psychologist has always made sure that the data gathered from his clinical observations conforms to the principles of natural science.

Still, specific mechanisms which, probably, lay at the core of synchronicity phenomena became a subject of discussion only in the early 1980 s thanks to the progress in experimental studies of certain concepts of quantum physics. In one of the relatively early papers devoted to this matter (Keutzer 1982), the synchronicity phenomenon was juxtaposed with the 'morphic resonance' hypothesis suggested by Rupert Sheldrake. In turn, the Sheldrake's theory is interpreted in the above study in the context of quantum non-locality, a consequence of solution of the so-called Einstein-Podolsky-Rosen paradox. In his later papers (Keutzer 1984a, b), this author clearly associates quantum non-locality with Jung's synchronicity. Afterwards, other researchers (Mansfield and Spiegelman 1989) have reviewed non-local quantum correlations, the Schrodinger's cat paradox, and experiments verifying Bell's inequalities in relation to the synchronicity phenomenon. The same researchers also reviewed the principle of superposition within the context of attempts to explain the synchronicity phenomenon (Mansfield and Spiegelman 1991). Furthermore, Mansfield, reviewing Jung's theory, has analyzed, among other aspects, the role of Bell's inequalities per se in respect to the problems of analytical psychology (Mansfield 1991). A similar paper (Germine 1991) also dealing with the synchronicity phenomenon, the concept of quantum non-locality, and the EinsteinPodolsky-Rosen and Schrodinger's cat paradoxes, is devoted to determination of the nature of consciousness. Study of relation between quantum non-locality and Jung's synchronicity, and the matters concerning Bell's inequalities continued in later papers (Mansfield and Spiegelman 1996).

Publications discussing, in one way or another, the synchronicity phenomenon in relation to the quantum entanglement phenomenon have appeared during the past decade (Walach 1999; Walach and Römer 2000; Duch 2002; Milgrom 2002; Primas 2003; Stillfried and Walach 2006; Teodorani 2006). The latest papers in this sphere of study contain quite detailed research (Lucado et al. 2007; Carminati and Martin 2008; Martin et al. 2009; Martin et al. 2010).

At the same time, it should be noted that attempts to explain the synchronicity phenomenon by considering other physical mechanisms not directly related to quantum non-locality (Zabriskie 1995) are quite scarce.

It should also be mentioned that some authors look at the quantum entanglement phenomenon in regard to other aspects of Jung's theory without linking this phenomenon directly to the synchronicity phenomenon (Blutner and Hochnadel 2010; Conte et al. 2010).

Nevertheless, even though the probable role of quantum non-locality in realization of synchronicity phenomena is clearly emphasized in the above papers, the question of what physiological mechanism might be responsible for the existence of quantum entanglement between different human bodies has not been considered so far. The only exceptions in this respect are, perhaps, the studies by Michael Hyland, who also believes that the synchronicity phenomenon is caused by quantum entanglement (Hyland 2004a). The criterion enabling to consider that the mentioned corresponding physiological mechanism has been suggested is a description of necessary and sufficient conditions for occurrence of quantum entanglement between biological molecules of different people. Broadly speaking, these molecules may not be interconnected by quantum entanglement prior to mutual interaction. As quantum entanglement between biological molecules of different people may not exist per se, in the absence of once occurred special physical interaction, it is only this criterion that can be valid. We cannot generally assume that available quantum entanglement between molecules of different people is taken for granted. In one of his papers (Hyland 2004b), Hyland made an assumption that quantum entanglement may exist both between cells of the same human body and between different subjects. Apparently, this 
author came very close to the solution of this problem in his another paper (Hyland 2003a), where he not only assumes existence of quantum entanglement at DNA level within the same body, but also describes possible role of quantum entanglement in meiosis processes. It is quite possible that all that needs to be done is just one little step further - to expand this author's perception of existence of quantum entanglement at DNA level within the same body and its role in morphogenesis to the hypothesis of existence of quantum entanglement between DNAs of different bodies. As far as meiosis processes are concerned, Hyland regards the role of quantum entanglement exclusively as regulating the cell division. The fact that meiosis may represent a mechanism ensuring quantum entanglement between different bodies was left out by this author. In the meantime, solution of the problem of existence of non-local quantum correlations between different bodies, in particular, synchronicity phenomena, could be right there. The answer to this question will be proposed in this paper below.

\section{Study goal and hypothesis}

Synchronicity phenomena have been studied for quite a while, and not just by Jung but (a much lesser-known fact) by Sigmund Freud, the founder of psychoanalysis. His several papers corroborate this fact (Freud 1922, 1953, 1966). Naturally, the famous Austrian psychiatrist used somewhat different terminology, but in the essence, he studied phenomena of the same nature. A telling fact: in the same period, scientific community gained understanding of the nature of quantum phenomena which later were associated with the synchronicity phenomenon. We are talking about the socalled Einstein-Podolsky-Rosen paradox, study of which helped postulate existence of quantum non-locality and quantum entanglement, closely related to non-local quantum correlations.

As we know, the quantum entanglement means a quantum-mechanical phenomenon in which quantum state of two or more objects should be described in interrelation with each other, even if individual objects are spaced apart. As a result, correlations appear between physical properties of these objects.

The quantum entanglement phenomenon is also viewed at in relation to such notions as quantum coherence and quantum superposition. The principle of superposition in quantum physics will be discussed further in connection with the Schrodinger's cat paradox.

Nevertheless, this branch of theoretical physics began experiencing rapid development only during the past few decades. It can be explained, first of all, by emerging possibilities for experimental verification of violation of the so-called Bell's inequalities. It is also important to note that quantum entanglement, as follows from definition and the nature of this phenomenon, represents the most 'functional' (comparing to other physical mechanisms) instrument as far as attempts to interpret Jung's synchronicity are concerned. Yet, it still remains unclear how exactly the quantum entanglement may exist at the level of material carrier of consciousness, i.e. brain, and how the quantum entanglement may ensure nonlocal correlations between different subjects which make synchronicity possible. Solution of this problem is the goal of the study this paper is devoted for.

Let's assume that correlation of mental processes in different people not exchanging any information among themselves, i.e. synchronicity, is caused by non-local quantum correlations (quantum entanglement) between certain parts of these people's brain (Persinger et al. 2008). At this point, it needs to be noted that we can't talk about existence of quantum entanglement as such between macroscopic objects. The 'quantum entanglement' term is applicable exclusively to the objects of microworld, particularly at submolecular level: molecular orbitals (electron shells) of molecules, chromophore parts, etc. Therefore, it is, for example, correct to say 'quantum entanglement between neuron structures at submolecular level' instead of 'quantum entanglement between nerve cells', or make the following formulation: 'quantum entanglement between electron shells of neuron molecules of different people' instead of saying 'quantum entanglement between different people'. Also, as of today, direct 
observation of effects involving quantum entanglement at the level of macroscopic objects is highly questionable. Nevertheless, it should be noted in this respect that by this time, certain attempts were made at experimental observation of these phenomena as part of the quantum superposition studies (Gevaux 2010; O'Connell et al. 2010). And finally, it is important to emphasize that quantum entanglement cannot directly represent a mechanism of communicating information per se. Quantum entanglement may serve only as an instrument of ensuring correlation of certain physical quantities. In this case, correlation may be implemented at indefinitely large distances without limitation on speed imposed by the special theory of relativity.

Currently, existence of quantum coherence and quantum entanglement in biological molecules is intensively studied and is considered proved at experimental level (Gilmore and McKenzie 2005; Plenio and Huelga 2008; Thorwart et al. 2009; Hossein-Nejad and Scholes 2010; Sarovar et al. 2010). Many researchers believe that quite specific problems in living organisms may be solved using quantum entanglement (Cai et al. 2010). However, when studying the synchronicity phenomenon it is important to find out how quantum entanglement between biological structures of different organisms may occur. In this respect, let's assume that quantum entanglement between biological molecules may occur as a result of 'coherent resonance energy transfer' (Jang et al. 2008; OlayaCastro et al. 2008; Collini and Scholes 2009; Nazir 2009; Kekovic et al. 2010; Nalbach et al. 2010; Scholes 2010). Some authors describe possibility of quantum entanglement occurring during electrostatic (Coulomb) interaction (Mishima et al. 2004). Consequently, one may also assume that quantum entanglement between parts of biological molecules occurs as a result of the above interaction. Another physical mechanism by virtue of which quantum entanglement can arise is the Fermi resonance (Xi-Wen and Chuan-Ming 2009; Peng and Hou 2010; Hou et al. 2010). However, it is obvious that mere presence of different people close to each other hardly offers a sufficient condition for occurrence of quantum entanglement between any biological structures of their bodies. Also, synchronicity phenomena (correlation of mental processes in different subjects) may, generally speaking, be observed even if these people have never communicated with each other. Therefore, it seems prudent to put forth a hypothesis, whereby quantum entanglement occurs, and is subsequently maintained, at the level of genetic material.

In addition to other studies of quantum coherence and quantum entanglement in biological molecules, possibility of these phenomena existing in nucleic acids, particularly in DNA, was also intensively studied in recent years (Ogryzko 1997; McFadden and Al-Khalili 1999; Bieberich 2000; Schempp 2003; Sirakoulis et al. 2004; Tulub and Stefanov 2007; Ogryzko 2008; Cooper 2009a, b). Analysis of development trends in this area of study allows to assume that this direction is very promising (Curtis and Hurtak 2004; Ananthaswamy 2010). It has been, for example, suggested that quantum effects may be responsible for morphogenesis processes (Hyland 2003b), and represent, in a way, a link between genotype and phenotype (Rosen 1996). Therefore, it is possible that DNAs of different cells within the same organism may be connected by quantum entanglement as a result of division of cell during mitosis. On the other hand, it is also fair to assume that quantum entanglement between DNAs of different cells may occur during meiosis, when gametes are forming. This situation may take place, for example, during homologous genetic recombination - crossing-over. It means that quantum entanglement may exist between cell structures of different organisms as well. There is, however, one important circumstance worth noting: theoretically, quantum entanglement between DNAs of brain cells may occur exclusively during embryogenesis. The explanation is as follows: as we know, nerve cells experience mitotic division only during prenatal period. During postnatal period, neurons do not divide. Small percentage of neural stem cells may be disregarded. As of today, several papers have been already devoted to the matters concerning quantum entanglement at the level of central nervous system cells (Pereira 2007; Pereira and 
Furlan 2009; Pereira and Furlan 2010). At the same time, it should be noted that quantum entanglement may exist not only between nuclear DNAs of different cells. Any cellular structures interacting both before and during mitosis and meiosis processes, and afterwards, after cells have divided, ending up in different cells, may potentially represent elements which cause existence of quantum entanglement between molecules of different cells (Hameroff 2004; Tulub 2004). It is hardly possible to describe existence of quantum entanglement between biological molecules of different organisms without taking into account mitosis and meiosis processes. It is so because in order that quantum entanglement does occur between the objects of the microworld, such objects should have interacted. Generally speaking, in case no interaction took place, the objects of the microworld will not be related with quantum entanglement. Some particular cases of quantum entanglement among fermions make an exception to this rule (Zhou 2000; Vedral 2003; Oh and Kim 2004; Clark et al. 2005; Vertesi 2007; Jie and Shi-Qun 2008; Habibian et al. 2010). However, for these rare instances it is characteristic to adhere to a series of specific conditions. At this stage of science development it is premature to suggest that such conditions exist as applied to neurones of several different people. Furthermore, another important question requires our attention. It concerns not the quantum entanglement occurrence mechanism, but the problem of its long-term preservation. We are talking about the so-called decoherence, a process which involves destruction of quantum coherence under impact of electromagnetic fields and other factors. Decoherence involves transformation of states characterized by quantum superposition (the so-called 'pure' states) into states where quantum phenomena cannot be observed ('mixed' states). Nevertheless, recently this problem has also been tackled at with increasing success (Shabani and Lidar, 2005; Manfredi and Hervieux 2006; Grace at al., 2007), particularly in terms of understanding the processes occurring in DNA (Ogryzko, 2008; Cooper, 2009a, b). And finally, we still have to find out how quantum entanglement may occur between cellular structures of quite large number of subjects, because during meiosis, genetic materials may be transferred to descendants only. A hypothesis may come to rescue, whereby all humans originated from the same center in Africa approximately 80-200 thousand years ago - 'recent single-origin hypothesis' (Batzer et al. 1994; Armour et al. 1996; Liu et al. 2006; Pritchard et al. 1996). Not extending, surely, the assumption of existence of quantum entanglement onto the scale of genome of all people, one may, nevertheless, assume that certain population groups bear in their genetic material DNA parts inherited from prehistoric men, which are common to these groups (Goldstein et al. 1995; Jorde et al. 1995; Nei and Takezaki 1996; Hey 1997). Therefore, existence of quantum entanglement between DNA molecules of relatively large groups of people cannot be ruled out.

Considering the aforementioned hypothesis, we can assume that 'material carriers of consciousness' include, in particular, molecular orbitals (electron shells) of molecules, biologically active during meiosis and mitosis. This conclusion is helped by phenomenology of analytical psychology, namely the synchronicity phenomenon.

An assumption that the molecules participating in meiosis can play a role of tangible media of consciousness, apart of the other biological molecules, by no means contradicts our formed presentation that the human psyche is 'localized' in the brain. An absence of such contradiction is conditional on the circumstance that the molecules displaying biological activity during meiosis are viewed merely as an 'intermediate' link when quantum entanglement is formed between brain cell molecules of various people. The molecules participating in a cell division during meiosis may appear as 'intermediaries' while the quantum entanglement is formed between certain brain cell molecules of a person with the same molecules of the brain cells belonging to another person. In its turn, biological molecules used during mitosis can be similar 'intermediaries' in the course of quantum entanglement formation. However, the molecules used during mitosis can ensure a formation of quantum entanglement not between molecules of various people but 
between the cell molecules of one human organism. Specifically, it can occur between the brain cell molecules and the molecules which are active when forming gametes of one and the same person. As it has already been noted, quantum entanglement between the molecules of brain neurons of an organism may, theoretically, arise during embryogenesis exclusively. Quantum entanglement between the brain cell molecules and the molecules of other cells within one and the same organism may also arise exclusively in the process of prenatal development. Among other things, it also refers to quantum entanglement arising between the brain cell molecules and the cell molecules of other organs wherein meiosis takes place within the frame of one organism. Still, quantum entanglement between the cell molecules of the organs wherein meiosis within the frame of one organism takes place may occur not only during embryogenesis but also in the postnatal period.

Again, an assumption that genetic material can be a tangible medium of consciousness has been contemplated so far by a number of researchers (Dennis 2010; Tariq et al 2010) and is not viewed now as something remarkable. Recently there also appear papers (Vaas 1999; Molyneux 2010) which allow of a rather skeptical attitude to a stereotyped notion that the nature of a human psyche is conditioned by electric transfer of signal in the brain.

In this context it should be pointed out that orientation to quantum effects when trying to explain the nature of consciousness and, specifically, orientation to quantum entanglement phenomenon (including at the genetic material level) seems more than justified. To corroborate this thesis let us consider modern information technologies. Complexity of contemporary supercomputers and computer networks, a number of electronic links therein and the volume of transferred information may be currently comparable with certain similar indices applicable to describe human brain properties. However, as yet nobody saw (generally, it is hard to imagine that anybody could have observed such a thing) any technical device possessing consciousness which operating principle was based upon a transfer of electronic signals. It refers, inter alia, to technical devices developed within the framework of artificial intelligence studies. Therefore, it appears highly doubtful that the brain of a newborn, able to transfer electric pulses only, is an adequate medium of consciousness and can provide for a development of the personality possessing intelligence. It seems so that the brain molecules of an individual are to be linked, via a genetic material and by means of quantum entanglement, with genetic material (and molecules at the level of the central nervous system) of a great number of other people.

Characteristically enough, a famous British biologist Rupert Sheldrake has come up with a similar hypothesis at his time, assuming that correlation of mental processes in different people is caused by DNA. At the same time, Sheldrake did not specify what physical mechanism precisely may be responsible for this correlation. And only some other authors have reviewed the Sheldrake's theory in light of quantum nonlocality (Keutzer 1982; Resconi and Nikravesh 2008). Also, key provisions of analytical psychology were viewed at in connection with David Bohm's theory of holomovement and Karl Pribram's holographic brain theory (Zinkin 1987). Besides Jung, the assumption that mental processes somehow correlate with certain processes occurring beyond human brain was put forth at the time by John Eccles, laureate of the 1963 Nobel Prize in Physiology (Popper and Eccles 1977) jointly with Wilder Penfield (Penfield 1978).

The hypothesis outlined in this article may be viewed at a different angle. As we know, phenomena which quantum mechanics deals with in no way fit the perception of surrounding world which we carry in everyday life. This is also true about the principle of superposition in quantum theory, a phenomenon when, for example, an object of microworld may be 'located' in several points of Hilbert space 'simultaneously' (Garraway and Knight 1994; Haroche et al. 1997; Deléglise et al. 2008). Back in his time, one of the founders of quantum theory Erwin Schrödinger showed that as a result of this principle, one can model a situation when a living creature, for example a cat, may be both 'alive' and 'dead' at the same time. However strange this 
thought experiment might seem to us, at this time practical experiments aimed at realization of quantum superposition at macroscopic level are already underway (Gevaux 2010; O'Connell et al. 2010). One of the consequences of existence of quantum entanglement between chromosomes of brain neurons in different people may be the fact that consciousness is not 'localized' in brain of an individual but, in the essence, 'simultaneously' 'belongs' to a group of people. This point of view is rather closer to Arnold Mindell's transpersonal interpretation (Mindell 2000, 2004) than, in fact, to the theory of collective unconscious.

\section{this study \\ Conclusions and prospects of}

\section{References}

Ananthaswamy A. Quantum entanglement holds together life's blueprint. New Scientist 2010; 207: 9-9.

Armour JA, Anttinen T, May CA, Vega EE, Sajantila A, Kidd $J R$, Kidd KK, Bertranpetit J, Pääbo $S$ and Jeffreys AJ. Minisatellite diversity supports a recent African origin for modern humans. Nature Genetics 1996; 13: 154160.

Batzer MA, Stoneking M, Alegria-Hartman M, Bazan $H$, Kass, DH, Shaikh TH, Novick GE, loannou PA, Scheer WD and Herrera RJ. African origin of human-specific polymorphic Alu insertions. Proceedings of the National Academy of Sciences 1994; 91: 1228812292.

Bieberich E. Probing quantum coherence in a biological system by means of DNA amplification. Biosystems 2000; 57: 109-124.

Blutner R and Hochnadel E. Two qubits for C.G. Jung's theory of personality. Cognitive Systems Research 2010; 11: 243-259.

Cai J, Guerreschi GG and Briegel HJ. Quantum control and entanglement in a chemical compass. Physical Review Letters 2010; 104: 220502.

Carminati GG and Martin F. Quantum mechanics and the psyche. Physics of Particles and Nuclei 2008; 39: 560577.

Clark SR, Alves CM and Jaksch D. Efficient generation of graph states for quantum computation. New Journal of Physics 2005; 7:124.

Collini E and Scholes GD. Coherent intrachain energy migration in a conjugated polymer at room temperature. Science 2009; 323: 369-373.

Conte E, Todarello O, Laterza V, Khrennikov AY, Mendolicchio $\mathrm{L}$ and Federici A. A preliminary experimental verification of violation of Bell inequality in a quantum model of Jung theory of personality formulated with Clifford algebra. Journal of Consciousness Exploration \& Research 2010; 1 : 831-849.
Progress in various areas of modern natural science allows to hope that Carl Jung's concept of synchronicity will, after all, receive scientific explanation. Surely, one shouldn't get carried away on a tide of euphoria, ecstatically accepting 'trendy' applications of the quantum entanglement phenomenon. Scientific practice implies experimental confirmation of hypotheses, not ruling out their disproof as well. In author's opinion, the hypothesis outlined in this article implies verification of currently available data to explain the nature of synchronicity phenomenon.
Cooper WG. Evidence for transcriptase quantum processing implies entanglement and decoherence of superposition proton states. Biosystems 2009a; 97: 73-89.

Cooper WG. Necessity of quantum coherence to account for the spectrum of time-dependent mutations exhibited by bacteriophage T4. Biochemical Genetics 2009b; 47: 892-910.

Curtis BD and Hurtak JJ. Consciousness and quantum information processing: uncovering the foundation for a medicine of light. Journal of Alternative and Complementary Medicine 2004; 10: 27-39.

Deléglise S, Dotsenko I, Sayrin C, Bernu J, Brune M, Raimond $\mathrm{J}$ and Haroche $\mathrm{S}$. Reconstruction of nonclassical cavity field states with snapshots of their decoherence. Nature 2008; 455: 510-514.

Dennis KL. Quantum Consciousness: Reconciling Science and Spirituality Toward Our Evolutionary Future(s). World Futures 2010; 66: 511-524.

Duch W. Synchronicity, mind, and matter. The International Journal of Transpersonal Studies 2002; 21: 153-168.

Freud S. Dreams and telepathy. International Journal of Psycho-Analysis 1922; 3: 283-305.

Freud S. 'Psychoanalysis and telepathy'. In Psychoanalysis and the Occult, Edited by George Devereux. New York: International Universities Press, Inc., 56-68, 1953.

Freud S. Lecture 30: 'Dreams and occultism' In Psychoanalysis and the Occult, Edited by George Devereux. New York: Norton, 91-109, 1966.

Garraway BM and Knight PL. Evolution of quantum superpositions in open environments: Quantum trajectories, jumps, and localization in phase space. Physical Review A 1994; 50: 2548-2563.

Germine M. Consciousness and synchronicity. Medical Hypotheses 1991; 36: 277-283.

Gevaux D. Quantum resonators: At the limit, at last. Nature Physics 2010; 6: 243-243. 
Gilmore J and McKenzie RH. Spin boson models for quantum decoherence of electronic excitations of biomolecules and quantum dots in a solvent. Journal of Physics: Condensed Matter 2005; 17: 1735.

Goldstein DB, Linares AR, Cavalli-Sforza LL and Feldman MW. Genetic absolute dating based on microsatellites and the origin of modern humans. Proceedings of the National Academy of Sciences 1995; 92: 6723-6727.

Grace M, Brif C, Rabitz H, Walmsley IA, Kosut RL and Lidar DA. Optimal control of quantum gates and suppression of decoherence in a system of interacting two-level particles. Journal of Physics B: Atomic, Molecular and Optical Physics 2007; 40: S103.

Habibian H, Clark JW, Behbood N and Hingerl K. Greenberger-Horne-Zeilinger and $W$ entanglement witnesses for the noninteracting Fermi gas. Physical Review A 2010; 81:032302.

Hameroff SR. A new theory of the origin of cancer: quantum coherent entanglement, centrioles, mitosis, and differentiation. Biosystems 2004; 77: 119-136.

Haroche S, Brune M. and Raimond JM. Experiments with single atoms in a cabity: entanglement, Schrödinger's cats and decoherence. Philosophical Transactions of the Royal Society of London A 1997; 355: 2367-2380.

Hey J. Mitochondrial and nuclear genes present conflicting portraits of human origins. Molecular Biology and Evolution 1997; 14: 166-172.

Hossein-Nejad $H$ and Scholes GD. Energy transfer, entanglement and decoherence in a molecular dimer interacting with a phonon bath. New Journal of Physics 2010; 12: 065045.

Hou X, Wan M and Ma Z. Entropy, energy and negativity in Fermi-resonance coupled states of substituted methanes. Journal of Physics A: Mathematical and Theoretical 2010; 43:205301.

Hyland ME. Extended network generalized entanglement theory: Therapeutic mechanisms, empirical predictions, and investigations. Journal of Alternative and Complementary Medicine 2003a; 9: 919-936.

Hyland ME. A brief guide to extended network entanglement theory as a theory of healing and its empirical predictions. Research in Complementary and Classical Natural Medicine 2003b; 10: 201-206.

Hyland ME. Emergent, entanglement, love and being. Journal of Holistic Healthcare 2004a; 1: 24-29.

Hyland ME. Does a form of 'entanglement' between people explain healing? An examination of hypotheses and methodology. Complementary Therapies in Medicine 2004b; 12: 198-208.

Jang S, Cheng Y, Reichman DR and Eaves JD. Theory of coherent resonance energy transfer. Journal of Chemical Physics 2008; 129: 101104.

Jie $R$ and Shi-Qun Z. Attack-Induced Entanglement of Noninteracting Fermi Gas. Communications in Theoretical Physics 2008; 49:1439.

Jorde LB, Bamshad MJ, Watkins WS, Zenger R, Fraley AE, Krakowiak PA, Carpenter KD, Soodyall H, Jenkins T and Rogers AR. Origins and affinities of modern humans: a comparison of mitochondrial and nuclear genetic data. The American Journal of Human Genetics 1995; 57: 523-538.
Kekovic G, Rakovic D, Tošic B, Davidovic D and Cosic I. Quantum foundations of resonant recognition model. Acta Physica Polonica A 2010; 117: 756-759.

Keutzer CS. Archetypes, synchronicity and the theory of formative causation. Journal of Analytical Psychology 1982; 27: 255-262.

Keutzer CS. Synchronicity in psychotherapy. Journal of Analytical Psychology 1984a; 29: 373-381.

Keutzer CS. The power of meaning: From quantum mechanics to synchronicity. Journal of Humanistic Psychology 1984b; 24: 80-94.

Liu H, Prugnolle F, Manica A and Balloux F. A geographically explicit genetic model of worldwide human-settlement history. The American Journal of Human Genetics 2006; 79: 230-237.

Lucado W, Römer $\mathrm{H}$ and Walach $\mathrm{H}$. Synchronistic phenomena as entanglement correlations in generalized quantum theory. Journal of Consciousness Studies 2007; 14: 50-74.

Manfredi $G$ and Hervieux PA. Loschmidt echo in a system of interacting electrons. Physical Review Letters 2006; 97: 190404.

Mansfield V and Spiegelman JM. Quantum mechanics and Jungian psychology: building a bridge. Journal of Analytical Psychology 1989; 34: 3-31.

Mansfield $\mathrm{V}$ and Spiegelman JM. The opposites in quantum physics and Jungian psychology: Part I: theoretical foundations. Journal of Analytical Psychology 1991; 36: 267-288.

Mansfield V. The opposites in quantum physics and Jungian psychology: Part II: applications. Journal of Analytical Psychology 1991; 36: 289-306.

Mansfield V and Spiegelman JM. On the physics and psychology of the transference as an interactive field. Journal of Analytical Psychology 1996; 41: 179-202.

Martin F, Carminati F and Carminati GG. Synchronicity, quantum information and the psyche. Journal of Cosmology 2009; 3: 580-589.

Martin F, Carminati F and Carminati GG. Quantum information, oscillations and the psyche. Physics of Particles and Nuclei 2010; 41: 425-451.

McFadden J and Al-Khalili J. A quantum mechanical model of adaptive mutation. Biosystems 1999; 50: 203-211.

Milgrom LR. Patient-practitioner-remedy (PPR) entanglement. Part 1 : a qualitative, non-local metaphor for homeopathy based on quantum theory. Homeopathy 2002; 91: 239-248.

Mindell A. Quantum Mind: the Edge Between Physics and Psychology. Portland, OR: Lao Tse Press, 2000.

Mindell A. The Quantum Mind and Healing: How to Listen and Respond to Your Body's Symptoms. Charlottesville, VA: Hampton Roads, 2004.

Mishima K, Hayashi $M$ and Lin SH. Entanglement in scattering processes. Physics Letters A 2004; 333: 371-377.

Molyneux B. Why the neural correlates of consciousness cannot be found. Journal of Consciousness Studies 2010; 17: 168-188.

Nalbach P, Eckel J and Thorwart M. Quantum coherent biomolecular energy transfer with spatially correlated fluctuations. New Journal of Physics 2010; 12: 065043. 
Nazir A. Correlation-dependent coherent to incoherent transitions in resonant energy transfer dynamics. Physical Review Letters 2009; 103: 146404.

Nei $\mathrm{M}$ and Takezaki $\mathrm{N}$. The root of the phylogenetic tree of human populations. Molecular Biology and Evolution 1996; 13: 170-177.

O'Connell AD, Hofheinz M, Ansmann M, Bialczak RC, Lenander $M$, Lucero $E$, Neeley $M$, Sank $D$, Wang $H$, Weides M, Wenner J, Martinis JM and Cleland AN. Quantum ground state and single-phonon control of a mechanical resonator. Nature 2010; 464: 697-703.

Ogryzko VV. A quantum-theoretical approach to the phenomenon of directed mutations in bacteria. Biosystems 1997; 43: 83-95.

Ogryzko VV. Erwin Schroedinger, Francis Crick and epigenetic stability. Biology Direct 2008; 3: 15.

Oh $\mathrm{S}$ and Kim J. Entanglement of electron spins of noninteracting electron gases. Physical Review A 2004; 69:054305.

Olaya-Castro A, Lee CF, Olsen FF and Johnson NF. Efficiency of energy transfer in a light-harvesting system under quantum coherence. Physical Review $B$ 2008; 78: 085115.

Penfield W. The Mystery of the Mind. Princeton: Princeton University Press, 1978.

Peng $D$ and Hou X. Fidelity and Mutual Entropy in Mixed States for Fermi-resonance Coupling Vibrations of CS2. Chinese Journal of Chemical Physics 2010; 23:393.

Pereira AJ. Astrocyte-trapped calcium ions: the hypothesis of a quantum-like conscious protectorate. Quantum Biosystems 2007; 1: 80-92.

Pereira AJ and Furlan FA. On the role of synchrony for neuron-astrocyte interactions and perceptual conscious processing. Journal of Biological Physics 2009; 35: 465-480.

Pereira AJ and Furlan FA. Astrocytes and human cognition: Modeling information integration and modulation of neuronal activity. Progress in Neurobiology 2010; 92: 405-420.

Persinger MA, Tsang EW, Booth $\mathrm{JN}$ and Koren SA. Enhanced power within a predicted narrow band of theta activity during stimulation of another by circumcerebral weak magnetic fields after weekly spatial proximity. NeuroQuantology 2008; 6: 7-21.

Plenio MB and Huelga SF. Dephasing-assisted transport: quantum networks and biomolecules. New Journal of Physics 2008; 10: 113019.

Popper KR and Eccles JC. The Self and Its Brain. Berlin, Heidelberg, London, New York: Springer International, 1977.

Primas $\mathrm{H}$. Time-entanglement between mind and matter. Mind and Matter 2003; 1: 81-119.

Pritchard JK, Feldman MW, Risch N, Kidd KK and Tishkoff SA. Genetic data and the African origin of humans. Science 1996; 274: 1548-1549.

Resconi G and Nikravesh M. Morphic computing. Applied Soft Computing 2008; 8: 1164-1177.

Rosen R. Biology and the measurement problem. Computers \& Chemistry 1996; 20: 95-100.

Sarovar M, Ishizaki A, Fleming GR and Whaley KB. Quantum entanglement in photosynthetic light- harvesting complexes. Nature Physics 2010; 6: 462 467.

Schempp W. Replication and transcription processes in the molecular biology of gene expressions: control paradigms of the DNA quantum holographic information channel in nanobiotechnology. Biosystems 2003; 68: 119-145.

Shabani A and Lidar DA. Theory of initialization-free decoherence-free subspaces and subsystems. Physical Review A 2005; 72: 042303.

Scholes GD. Quantum-coherent electronic energy transfer: Did nature think of it first?. Physical Chemistry Letters 2010; 1: 2-8.

Sirakoulis GC, Karafyllidis I, Sandaltzopoulos R, Tsalides P and Thanailakis A. An algorithm for the study of DNA sequence evolution based on the genetic code. BioSystems 2004; 77: 11-23.

Stillfried $\mathrm{N}$ and Walach $\mathrm{H}$. The whole and its parts: Are complementarity and non-locality intrinsic to closed systems?. International Journal of Computing Anticipatory Systems 2006; 17: 137-146.

Tariq S, Kazim R and Tauqir I. Could the human mind be a product of mental genes: a nonbiological component of brain genes ? NeuroQuantology 2010; 8: 359-377.

Teodorani M. Synchronicity - The Link Between Physics and Psyche, from Pauli and Jung to Chopra. Cesena, 2006.

Thorwart M, Eckel J, Reina JH, Nalbach P and Weiss S. Enhanced quantum entanglement in the nonMarkovian dynamics of biomolecular excitons. Chemical Physics Letters 2009; 478: 234-237.

Tulub AA. Coherent triplet and singlet states in tubulin dynamics. Future Generation Computer Systems 2004; 20: 773-780.

Tulub AA and Stefanov VE. Triplet-singlet spin communication between DNA nucleotides serves the basis for quantum computing. Chemical Physics Letters 2007; 436: 258-262.

Vaas R. Why neural correlates of consciousness are fine, but not enough. Anthropology \& Philosophy 1999; 3: 121-141.

Vedral V. Entanglement in the second quantization formalism. Central European Journal of Physics 2003; 1:289-306.

Vertesi T. Genuine tripartite entanglement in the noninteracting Fermi gas. Physical Review A 2007; 75:042330.

Walach H. Magic of signs: A nonlocal interpretation of homeopathy. Journal of Scientific Exploration 1999; 13: 291-315.

Walach H. and Römer H. Complementarity is a useful concept for consciousness studies. A Reminder. Neuroendocrinology Letters 2000; 21: 221-232.

$\mathrm{Xi}-$ Wen $\mathrm{H}$ and Chuan-Ming C. Dynamical entanglement for Fermi coupled stretching and bending modes. Chinese Physics B 2009; 18:2719.

Zabriskie B. Jung and Pauli. A subtle asymmetry. Journal of Analytical Psychology 1995; 40: 531-553.

Zhou T. An evolution from quantum entanglement to classical disentanglement and its consequences in statistical mechanics. Solid State Communications 2000; 115:185-189. 
Zinkin L. The Hologram as a model for analytical psychology. Journal of Analytical Psychology 1987; 32: $1-21$. 